\title{
DOBLE CAUSA DE CEGUERA: CORIORRETINITIS ESCLOPETARIA Y HEMIANOPSIA HOMÓNIMA
}

\section{DUAL CAUSE OF BLINDNESS: CHORIORETINITIS SCLOPETARIA AND HOMONYMOUS HEMIANOPSIA}

\author{
PÉREZ-CARRO G ${ }^{1}$, JUNCEDA-MORENO C ${ }^{1}$
}

\begin{abstract}
RESUMEN
Caso clínico: Se describe el caso de un varón que tras una agresión con arma de fuego en la región facial derecha presenta una coriorretinopatía esclopetaria (CS) en ojo derecho y una heminanopsia homónima contralateral.

Discusión: La CS es una rara entidad producida por la lesión ocular por una bala al atravesar la órbita. Además es rara encontrarla asociada a una alteración campimétrica causada por el trayecto y el alojamiento de la misma bala en el cerebro.
\end{abstract}

Palabras clave: Coriorretinitis esclopetaria, hemianopsia homónima, arma de fuego.

\begin{abstract}
Clinical case: We describe the case of a man who, after a gunshot wound to the right facial region, exhibited chorioretinitis sclopetaria of the right eye and a contralateral homonymous hemianopsia.

Discussion: Chorioretinitis sclopetaria is a rare entity resulting from an ocular injury caused by a bullet passing through the orbit. It is not common to find the condition associated with a damaged visual field caused by the subsequent path traversed and lodging of that bullet in the brain (Arch Soc Esp Oftalmol 2006; 81: 119-122).
\end{abstract}

Key words: Chorioretinitis sclopetaria, Homonymous hemianopsia, firearm.

\section{INTRODUCCIÓN}

La coriorretinopatía esclopetaria (CS) es una manifestación poco común de un traumatismo ocular no penetrante, secundario al trayecto de un proyectil entre el globo ocular y las paredes de la órbita (1-5). Se propone un doble mecanismo de lesión, directo por el trayecto y otro indirecto por las ondas de choque transmitidas al globo ocular. Se presenta el caso de un paciente con esta lesión y las distintas teorías acerca de su etiopatogenia.

\section{CASO CLÍNICO}

Varón de 28 años de edad que había sufrido una agresión por arma de fuego. Tiene alojada una bala en región occipital derecha y presentaba crisis

Recibido: 4/3/05. Aceptado: 20/2/06

Servicio de Oftalmología. Hospital de Cabueñes. Gijón. Principado de Asturias. España.

1 Licenciado en Medicina.

Comunicación presentada en panel en el LXXX Congreso de la S.E.O. (Córdoba 2004).

2. ${ }^{\circ}$ Premio a la Comunicación en panel.

Correspondencia:

Gema Pérez Carro

C/. Velázquez, 2, 2. ${ }^{\circ} \mathrm{I}$

33211 Gijón (Principado de Asturias)

España

E-mail: gemurri27@yahoo.es 
comiciales por encefalomalacia temporal derecha (figs. 1 y 2). Fue enviado al Servicio de Oftalmología para valoración por pérdida de visión del ojo derecho. La agudeza visual (AV) del ojo derecho (OD) era menor de 0,02 y la del ojo izquierdo (OI) de la unidad. Los datos de motilidad ocular intrínseca, polo anterior y presión intraocular eran nor-

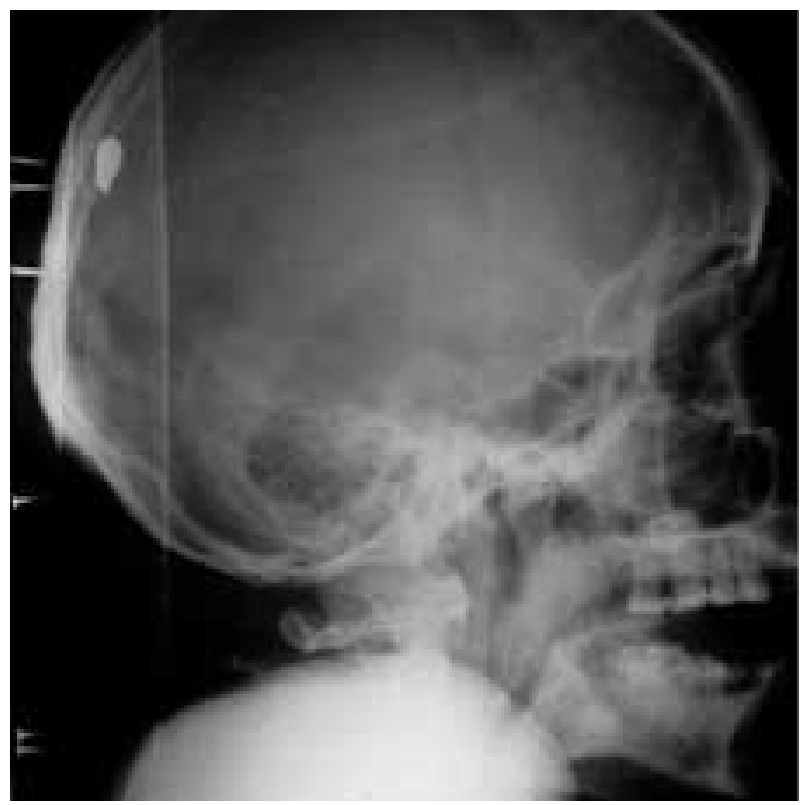

Fig. 1: Placa radiográfica lateral de cráneo donde se aprecia bala alojada en región occipital.
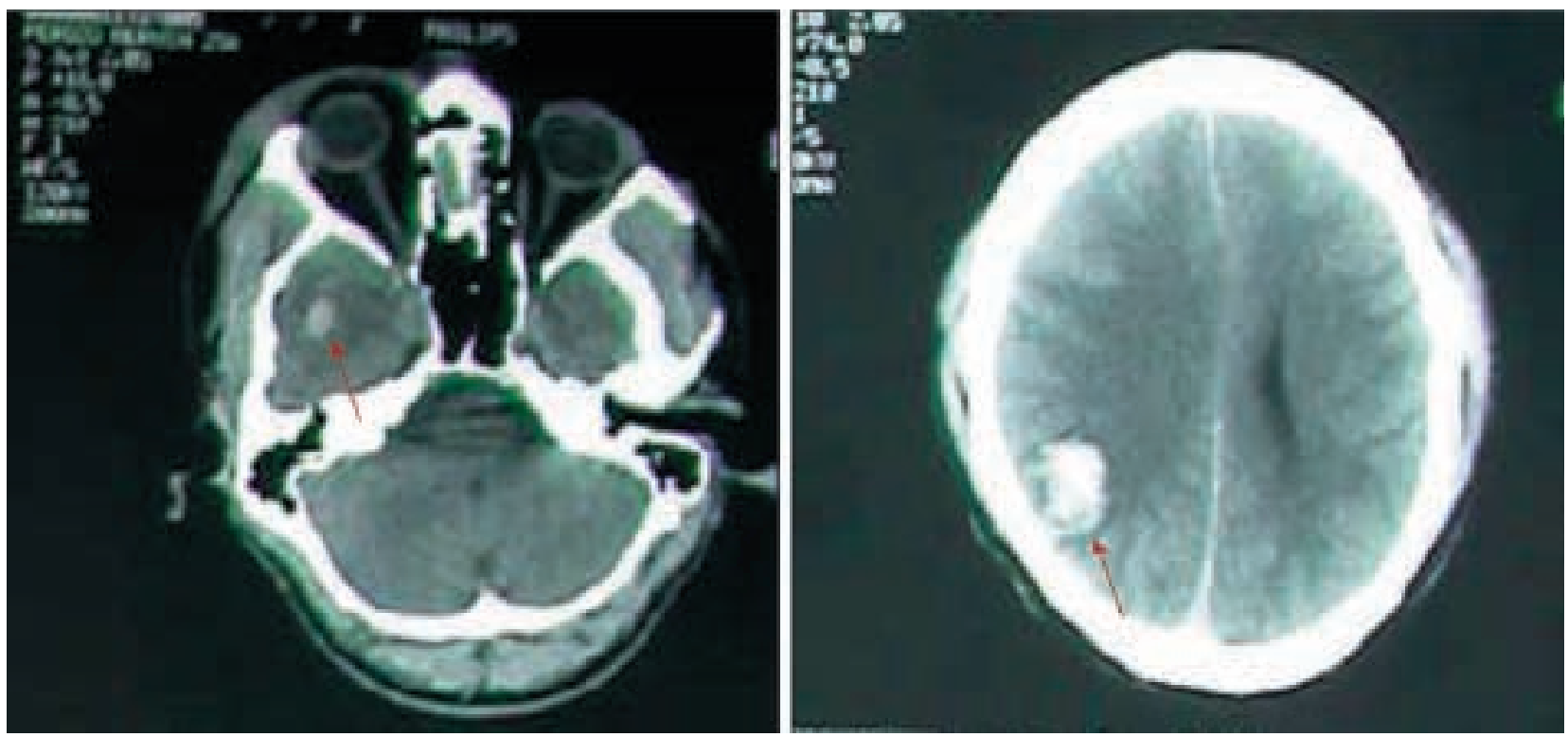

Fig. 2: Tc craneal: Encefalomalacia temporal derecha. males en ambos ojos. En la exploración del campo visual se evidenció una hemianopsia homónima izquierda (fig. 3).

En el fondo de ojo del OD presentaba una cicatriz coriorretiniana localizada en el polo posterior en la región temporal inferior con movilización de pigmento en el área macular y una membrana epirretiniana sin tracción, y la coloración de la papila es normal. El fondo de ojo del OI era normal (fig. 4). En la angiografía se apreció desde las fases iniciales una hiperfluorescencia apolillada y en parche en la zona de la rotura coroidea y retiniana, que no aumentaba en tamaño ni en intensidad, por la pérdida del epitelio pigmentario de la retina; ausencia de neovascularización subretiniana, una discreta tracción centrípeta vascular y una papila normal (fig. 5). En los potenciales evocados visuales (flash y pattern) se obtuvieron respuestas con aumento de latencia bilateral y una disminución de amplitud, hallazgos compatibles con una neuropatía axonal del II par. El EOG Y ERG no mostraban alteraciones.

\section{DISCUSIÓN}

La CS fue descrita como tal en 1901 por Goldhiezer en Alemania aunque ya otros autores la mencionan como una entidad, como Cohn en 1872 (14). Ha recibido otros nombres como ruptura corio- 

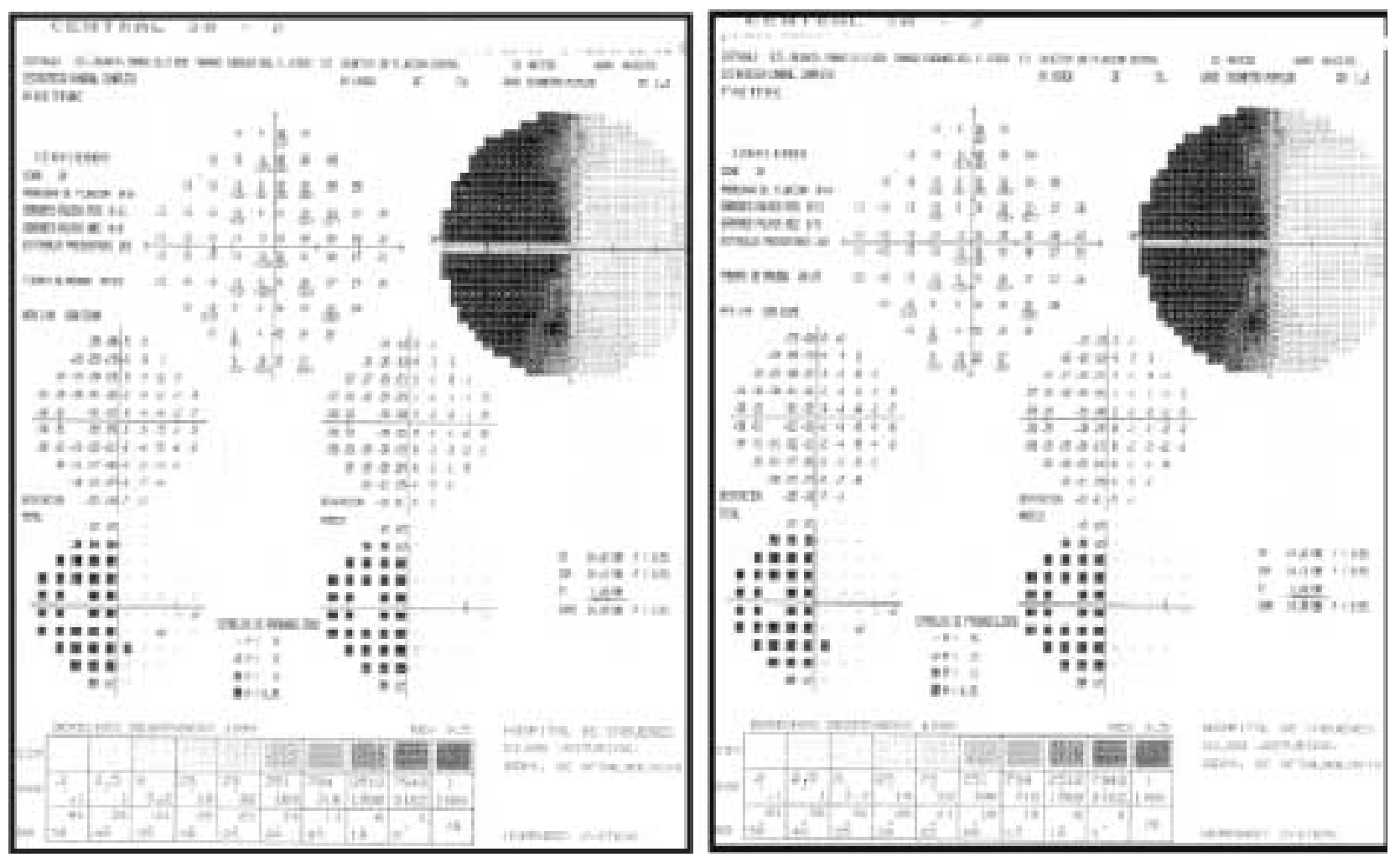

Fig. 3: Campo visual ( Humphrey 30/2):Hemianopsia homónima izquierda.

rretiniana traumática y coriorretinopatía proliferativa de Lagrange (1-3).

El origen etimológico de la palabra esclopetaria podría relacionarse con el verbo del inglés antiguo

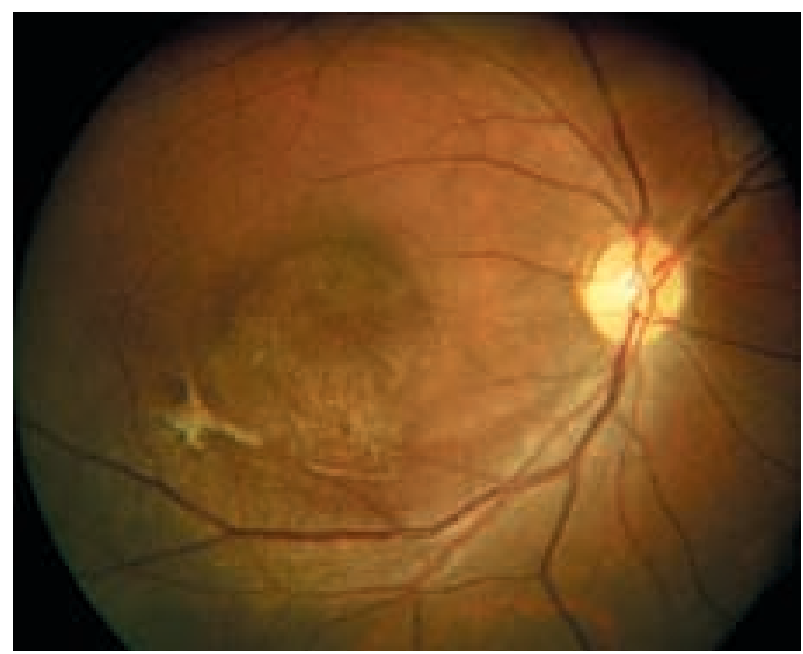

Fig. 4a: Fondo de ojo OD: Cicatriz coriorretiniana característica, bordes aserrados irregulares. Dispersión pigmentaria. «sclow» que significa desgarrar o rasgar o con una palabra del latín «sclopetum» que significa arma alargada italiana. El término de coriorretinitis haría referencia a la inflamación postraumática $(1,2)$.

La lesión se compone por una cicatriz coriorretiniana de bordes aserrados e hiperpigmentados (1-

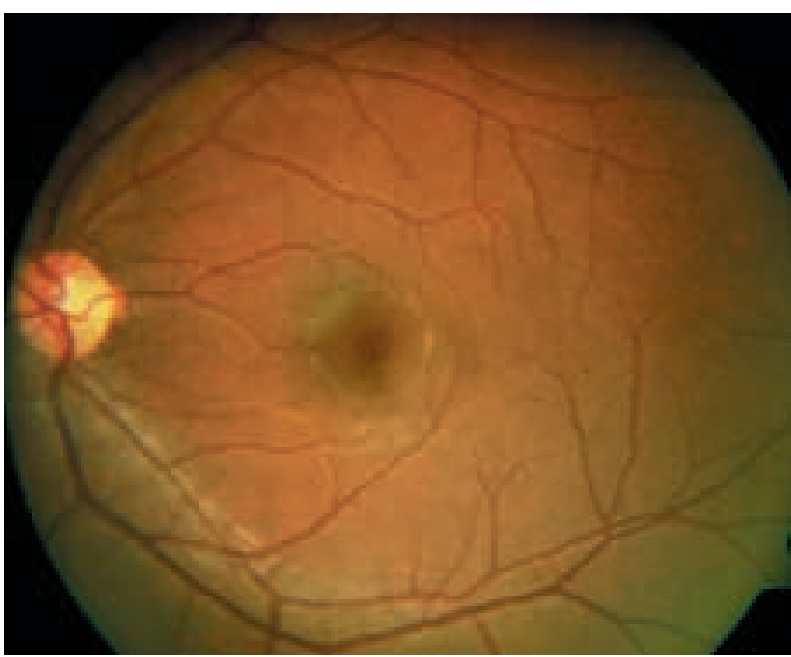

Fig. 4b: Fondo de ojo OI: Normal. 


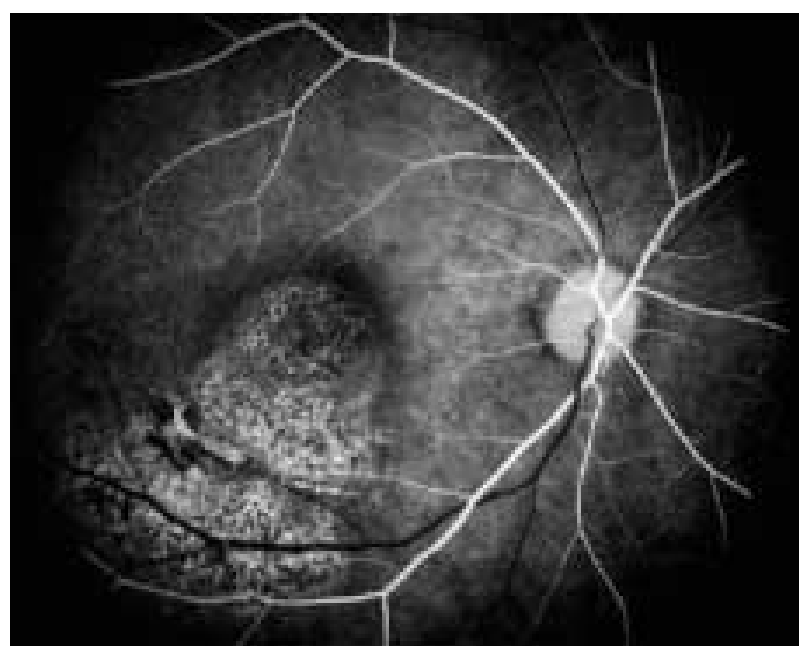

Fig. 5a: AFG. Hiperfluorescencia apolillada y en parche en tiempos precoces

5), membrana epirretiniana (MER), dispersión de pigmento en la mácula $(1,4)$ y daño en el nervio óptico.

Se produce por una ruptura simultánea y completa de las capas coroidea, retiniana y la membrana de Bruch (MB) dejando visible la esclera íntegra $(1,2)$, entendiendo que la lesión se origina por un mecanismo de disrupción mecánica y retracción tisular (2).

En cuanto a las posibles complicaciones se ha descrito el bajo riesgo de membrana neovascular subretiniana y de desprendimiento de retina $(2,3,5)$ en la zona de la ruptura, debido la integridad de la hialoides posterior (frecuente individuos jóvenes) y a la de la unión retina-coroides $(1,2)$. Sin embargo, podrían existir desgarros en la periferia lo que obliga a su seguimiento $(1,2)$.

El defecto de campo visual secundario a la lesión de la vía óptica intracerebral podría evaluarse mediante técnicas de neuroimagen seriadas viendo así el recorrido de la bala y la relación topográfica con sus secuelas. En este caso además es destacable la contraindicación para realizar una resonancia nuclear magnética.

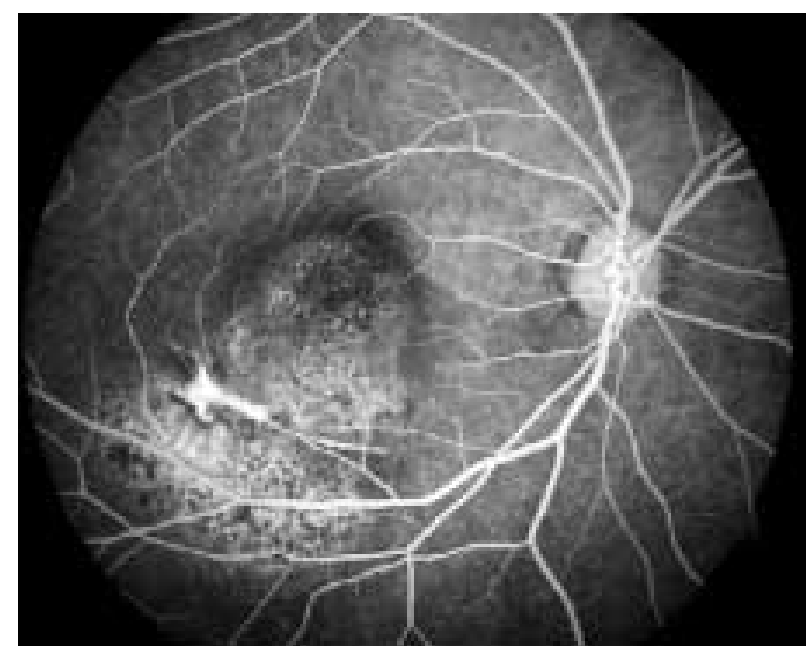

Fig. 5b: AFG. Ligero aumento en intensidad por relleno del defecto del EPR sin aumentar en tamaño en fases más tardías, no evidencia de MNNVSR.

En conclusión estamos ante una entidad poco común, en su mayor parte debida a agresiones por arma de fuego periorbitarias, con unos hallazgos específicos y con pocas complicaciones por su patogenia. Que además en el paciente a estudio se asocia a una lesión retrogeniculada de la vía nerviosa óptica que le ocasiona un defecto en el campo visual contralateral causante de su doble ceguera.

\section{BIBLIOGRAFÍA}

1. Dubovy SR, Guyton DL, Green WR. Clinicopathologic correlation of chorioretinitis sclopetaria. Retina 1997; 17: 510-520.

2. Martin DF, Awh CC, McCuen BW 2nd, Jaffe GJ, Slott JH, Machemer R. Treatment and pathogenesis of traumatic chorioretinal rupture (sclopetaria). Am J Ophthalmol 1994; 117: 190-200.

3. Richards RD, West CE, Meisels AA. Chorioretinitis sclopetaria. Am J Ophthalmol 1968; 66: 852-860.

4. Otto CS, Nixon KL, Mazzoli RA, Raymond WR 4th, Ainbinder DJ, Hansen EA, et al. Chorioretinitis sclopetaria from BB ex memoria. Ophthalmic Surg Lasers 2001; 32: 152-154.

5. Gass JD. Stereoscopic atlas of macular diseases. Diagnosis and treatment. St. Louis: Mosby; 1997; II: 742-743. 